\title{
"An analysis of a mean-variance enhanced index tracking problem with weights
}

constraints"

\begin{tabular}{|c|c|}
\hline AUTHORS & $\begin{array}{l}\text { Wanderlei Lima de Paulo (D https://orcid.org/0000-0001-5242-3230 } \\
\text { Marta Ines Velazco Fontova (D https://orcid.org/0000-0002-7685-1229 } \\
\text { Renato Canil de Souza }\end{array}$ \\
\hline ARTICLE INFO & $\begin{array}{l}\text { Wanderlei Lima de Paulo, Marta Ines Velazco Fontova and Renato Canil de } \\
\text { Souza (2018). An analysis of a mean-variance enhanced index tracking problem } \\
\text { with weights constraints. Investment Management and Financial Innovations, } \\
\text { 15(4), 183-192. doi:10.21511/imfi.15(4).2018.15 }\end{array}$ \\
\hline DOI & http://dx.doi.org/10.21511/imfi.15(4).2018.15 \\
\hline RELEASED ON & Monday, 19 November 2018 \\
\hline RECEIVED ON & Wednesday, 22 August 2018 \\
\hline ACCEPTED ON & Friday, 02 November 2018 \\
\hline LICENSE & $\begin{array}{l}(c) \text { EY } \\
\text { This work is licensed under a Creative Commons Attribution } 4.0 \text { International } \\
\text { License }\end{array}$ \\
\hline JOURNAL & "Investment Management and Financial Innovations" \\
\hline ISSN PRINT & $1810-4967$ \\
\hline ISSN ONLINE & $1812-9358$ \\
\hline PUBLISHER & LLC "Consulting Publishing Company "Business Perspectives" \\
\hline FOUNDER & LLC "Consulting Publishing Company "Business Perspectives" \\
\hline
\end{tabular}

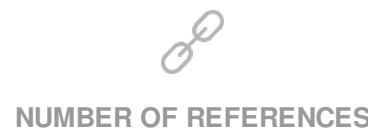

16
NUMBER OF FIGURES

3
NUMBER OF TABLES

4

(C) The author(s) 2023. This publication is an open access article. 


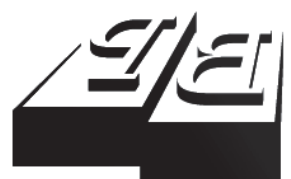

BUSINESS PERSPECTIVES

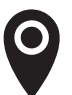

LLC "CPC "Business Perspectives" Hryhorii Skovoroda lane, 10, Sumy, 40022, Ukraine

www.businessperspectives.org

Received on: 22 $2^{\text {nd }}$ of August, 2018 Accepted on: $2^{\text {nd }}$ of November, 2018

(C) Wanderlei Lima de Paulo, Marta Ines Velazco Fontova, Renato Canil de Souza, 2018

Wanderlei Lima de Paulo, Ph.D., Professor of Quantitative Finance at Campo Limpo Paulista University Center, Campo Limpo Paulista, Brazil; University of Sao Paulo, São Paulo, Brazil.

Marta Ines Velazco Fontova, Ph.D., Professor of Operational Research at Campo Limpo Paulista University Center, Campo Limpo Paulista, Brazil.

Renato Canil de Souza, B.S., Student of Actuarial Science at University of Sao Paulo, Brazil.

\section{() (i)}

This is an Open Access article, distributed under the terms of the Creative Commons Attribution 4.0 International license, which permits unrestricted re-use, distribution, and reproduction in any medium, provided the original work is properly cited.
Wanderlei Lima de Paulo (Brazil), Marta Ines Velazco Fontova (Brazil),

Renato Canil de Souza (Brazil)

\section{AN ANALYSIS OF A MEAN- VARIANCE ENHANCED INDEX TRACKING PROBLEM WITH WEIGHTS CONSTRAINTS}

\begin{abstract}
In this paper, the authors deal with a mean-variance enhanced index tracking (EIT) problem with weights constraints. Using a shrinkage approach, they show that constructing the constrained EIT portfolio is equivalent to constructing the unconstrained EIT portfolio. This equivalence allows to study the effect of weights constraints on the covariance matrix and on the EIT portfolio. In general, the effects of weights constraints on the EIT portfolio are different from those observed in the case of global minimum variance portfolio. Finally, the authors present a numerical asset allocation example, where the S\&P 500 index is used as the market index to be tracked using a portfolio composed of ten stocks, in which the constrained EIT portfolio shows a satisfactory performance when compared to the unconstrained case.
\end{abstract}

Keywords

G10, G11
JEL Classification enhanced index track

\section{INTRODUCTION}

In general, an index tracking problem aims at establishing an optimal allocation so that the return of the portfolio replicates the return of a market index (passive management strategy), without purchasing all of the assets that compose the market index. On the other hand, the so-called enhanced index tracking (EIT) problem consists of constructing a portfolio that replicates and outperforms the market index by generating excess return. The literature presents different approaches and methods to construct an indexed portfolio, considering transaction cost, cardinality and weights constraints (see, for example, Canakgoz \& Beasley, 2008; Filippi et al., 2016; Paulo et al., 2016; Sant'Anna et al., 2017; Goel et al., 2018; Benidis et al., 2018).

Usually, the studies on tracking problem use the historical look-back approach, in which the tracking portfolio is constructed considering a sample of past observations. The classical uni-period mean-variance approach is considered in Roll (1992), Alexander and Baptista (2010) and Paulo et al. (2016), for example. Specifically, Paulo et al. (2016) studied an unconstrained EIT problem (e.g., allow short-selling), for which the authors derive an analytical solution. Differently from the cardinality constraint approach, they consider an approach in which the EIT portfolio is composed of a previously selected subset of assets belonging to the market index portfolio. Following the same approach, in this paper, we deal with a mean-variance EIT problem with weights constraints. In this case, it is not possible to obtain an analytic solution, but the problem can be easily solved using a quadratic programming algorithm. 
Particularly, the weights constraints play an important rule in the asset allocation problem, since it allows to control the level of short and long positions, as well as to avoid the concentration risk (when a portfolio has a large exposure to an asset or few assets), for example. In the work of Jagannathan and $\mathrm{Ma}$ (2003), the authors studied the effects of weights constraints on the global mean-variance portfolio using a shrinkage approach. Considering the same framework, we have shown that constructing a constrained mean-variance EIT portfolio is equivalent to constructing an unconstrained EIT portfolio (as studied in Paulo et al., 2016). This equivalence allows us to study the effect of weights constraints on the covariance matrix and on the EIT portfolio.

The remainder of the paper is organized as follows. Section 1 includes the formulation of the EIT problem with weights constraints and its optimality conditions. In section 2 , it is shown that constructing the constrained EIT portfolio is equivalent to constructing the unconstrained EIT portfolio using a shrinkage covariance matrix. A numerical example for an EIT portfolio using the S\&P 500 index as market index is presented in section 3. Final section presents some remarks.

\section{PROBLEM FORMULATION AND OPTIMALITY CONDITIONS}

We recall that an EIT problem aims at constructing a portfolio that replicates a market index and at the same time obtaining positive excess return (on average). In this case, this problem can be seen as a dual-objective optimization problem, a tradeoff between maximizing the mean excess return and minimizing a tracking error measure (measure of how closely a portfolio outperforms the index).

Let us consider a portfolio composed of $n$ assets (usually belonging to the market index). We denoteby $\omega=\left(\omega_{1} \ldots \omega_{n}\right)^{\prime}$ thevector with theweights of the assets in the portfolio, by $R=\left(R_{1} \ldots R_{n}\right)^{\prime}$ the random vector with the returns, of the assets and by $\sum=E\left((R-r)(R-r)^{\prime}\right)>0$ the covariance matrix of $R$, such that $r=E(R)$. Let $P_{e}$ be the error between the return obtained from the portfolio and the return obtained from the market index, so that $P_{e}=\omega^{\prime} R-R_{M}$, where $R_{M}$ is the return of the market index with $\mu_{M}=E\left(R_{M}\right)$. Then, the mean excess return and the tracking error (variance of the error $P_{e}$ ) are defined as $\mu_{e}=\omega^{\prime} r-\mu_{M}$ and $\sigma_{e}^{2}=\omega^{\prime} \Sigma \omega-2 \sigma_{M}^{2} \omega^{\prime} \beta+\sigma_{M}^{2}$, where $\beta=\left(\beta_{1} \ldots \beta_{n}\right)$ is the vector of asset betas and $\sigma_{M}^{2}$ is the variance of the market index. Based on the work of Paulo et al. (2016), we consider the following mean-variance EIT problem with weights constraints: minimize

$$
\frac{\varphi}{2}\left(\omega^{\prime} \Sigma \omega-2 \sigma_{M}^{2} \omega^{\prime} \beta+\sigma_{M}^{2}\right)-\left(\omega^{\prime} r-\mu_{M}\right),
$$

subject to

$$
\begin{aligned}
& e^{\prime} \omega=1, \\
& \omega_{i} \geq w_{i}^{-}, i=1, \ldots, n, \\
& \omega_{i} \leq w_{i}^{+}, i=1, \ldots, n,
\end{aligned}
$$

where $e$ represents a vector of ones of suitable dimension, $w_{i}^{-}$and $w_{i}^{+}$are the minimum and maximum proportion that must be held of asset $i$, respectively, so that $w_{i}^{-} \geq 0$ represents the condition in which the short-selling is not allowed (for $w_{i}^{-}<0$ short positions are allowed), and $\varphi>0$ is a trade-off parameter.

From constraints (3)-(4), it is possible to control the long and short positions in the portfolio. Other approaches have been proposed in the literature to consider short-selling constraints (see, for example, Kim et al., 2016; Fan et al., 2012; DeMiguel et al., 2009). Moreover, we recall that the objective of the problem (1)-(4) is to find an investment strategy $\omega$ that minimizes the tracking error $\left(\sigma_{e}^{2}\right)$ and maximizes the mean excess return $\left(\mu_{e}\right)$ simultaneously, where the trade-off between $\sigma_{e}^{2}$ and $\mu_{e}$ is balanced by the parameter $\varphi$. In general, by an appropriate choice of this parameter, a manager could define one of the three investment strategies (as in Paulo et al., 2016): i) achieve an average re $\theta$ turn rate higher than a market index (active man- 
agement strategy), ii) replicate the return rate of a market index (index tracking strategy) or iii) track a market index with a positive excess return rate (EIT strategy).

In the following, we present the optimality conditions for the constrained problem (1)-(4) and show that its solution solves the unconstrained problem (e.g., $\omega \in \mathfrak{R}^{n}$ ) using a shrinkage covariance matrix proposed by Jagannathan and Ma (2003). Studies related to the shrinkage covariance matrix estimators can be seen in Ledoit and Wolf (2004), DeMiguel et al. (2009) and Pantaleo et al. (2011), for example.

Setting $w^{-}=\left(w_{1}^{-} \ldots w_{n}^{-}\right)^{\prime}$ and $w^{+}=\left(w_{1}^{+} \ldots w_{n}^{+}\right)^{\prime}$, the Lagrange of the problem (1)-(4) is given by

$$
\begin{aligned}
& L(\omega, \lambda, \delta)=\frac{\varphi}{2}\left(\omega^{\prime} \Sigma \omega-2 \sigma_{M}^{2} \omega^{\prime} \beta+\sigma_{M}^{2}\right)- \\
& -\left(\omega^{\prime} r-\mu_{M}\right)-\tilde{\lambda}_{0}\left(e^{\prime} \omega-1\right)-\lambda^{\prime}\left(\omega-w^{-}\right)- \\
& -\delta^{\prime}\left(w^{+}-\omega\right),
\end{aligned}
$$

where $\lambda=\left(\lambda_{1} \ldots \lambda_{n}\right)^{\prime}$ are the Lagrange multipliers for the constraints (3), $\delta=\left(\delta_{1} \ldots \delta_{n}\right)^{\prime}$ are the Lagrange multipliers for the constraints (4) and $\tilde{\lambda}_{0}$ is the multiplier for the constraint (2), with $\lambda_{i} \geq 0$ and $\delta_{i} \geq 0$. In this case, the optimality conditions are given by:

$$
\begin{gathered}
\varphi\left(\Sigma \omega-\sigma_{M}^{2} \beta\right)-r-\tilde{\lambda}_{0} e-\lambda+\delta=0, \\
e^{\prime} \omega-1=0, \\
\lambda_{i}=0 \text { if } \omega_{i}>w_{i}^{-}, \\
\delta_{i}=0 \text { if } \omega_{i}<w_{i}^{+} .
\end{gathered}
$$

As the problems (1)-(4) are strictly convex quadratic optimization problem, the system (5)-(8) establishes the necessary and sufficient conditions for the existence of an optimal solution, so that if $\tilde{\omega}$ is a solution of the system, then it is a solution of the problems (1)-(4) and vice versa. In this case, it is not possible to obtain an analytic solution, but the solution can be obtained efficiently using quadratic programming solvers. On the other hand, Paulo et al. (2016) derive an analytical solution for the unconstrained problem $\left(\omega \in \mathfrak{R}^{n}\right)$, whose optimality conditions are given by

$$
\begin{gathered}
\varphi\left(\Sigma \omega-\sigma_{M}^{2} \beta\right)-r-\lambda_{0} e=0, \\
e^{\prime} \omega-1=0,
\end{gathered}
$$

where $\lambda_{0}$ is the Lagrange multiplier for the constraint $e^{\prime} \omega=1$, so that the optimal solution can be written as

$\omega^{*}=\Sigma^{-1}\left(\sigma_{M}^{2} \beta+\frac{r}{\varphi}+\frac{\Gamma}{\alpha} e\right)$

where $\quad \Gamma=1-\sigma_{M}^{2} e^{\prime} \Sigma^{-1} \beta-\left(e^{\prime} \Sigma^{-1} r\right) / \varphi \quad$ and $\alpha=e^{\prime} \Sigma^{-1} e$.

\section{SOME THEORETICAL RESULTS}

In the work of Jagannathan and Ma (2003), the authors have shown that constructing a constrained global minimum variance portfolio is equivalent to constructing an unconstrained minimum variance portfolio after modifying the covariance matrix in a particular way (shrinkage approach). In this section, following the same analysis and procedure as in Roncalli (2011) and Jagannathan and $\mathrm{Ma}$ (2003), we show the same equivalence for the mean-variance EIT problem (subsection 2.1) and we analyze the effects of weights constraints on the EIT portfolio (subsection 2.2).

\subsection{Shrinkage covariance matrix approach}

In the following proposition, we show that the solution of the constrained problem (1)-(4) solves the unconstrained problem (e.g., $\omega \in \mathfrak{R}^{n}$ ) using a shrinkage covariance matrix as proposed by Jagannathan and Ma (2003).

Proposition 1. Let us consider the shrinkage covariance matrix

$$
\tilde{\Sigma}=\Sigma+\frac{(\delta-\lambda) e^{\prime}}{\varphi}+\frac{e(\delta-\lambda)^{\prime}}{\varphi},
$$

where $\lambda$ and $\delta$ are the Lagrange multipliers for the problem (1)-(4), with $\varphi>0$. Assuming $\tilde{\Sigma}>0$, then the solution of the constrained problem (1)(4), called here $\tilde{\omega}$, is a solution of the unconstrained problem (e.g., $\omega \in \mathfrak{R}^{n}$ ) with $\Sigma=\tilde{\Sigma}$. 
Table 1. Elements of the perturbation matrix, $\Delta_{i, j}$, related to the optimized weights $\tilde{\omega}_{i}$ and $\tilde{\omega}_{j}$

\begin{tabular}{l|c|c|c} 
& $\tilde{\omega}_{j}=w_{j}^{-}$ & $w_{j}^{-}<\tilde{\omega}_{j}<w_{j}^{+}$ & $\tilde{\omega}_{j}=w_{j}^{+}$ \\
\hline$\tilde{\omega}_{i}=w_{i}^{-}$ & $-\left(\lambda_{i}+\lambda_{j}\right) / \varphi$ & $-\lambda_{i} / \varphi$ & $\left(-\lambda_{i}+\delta_{j}\right) / \varphi$ \\
\hline$w_{i}^{-}<\tilde{\omega}_{i}<w_{i}^{+}$ & $-\lambda_{j} / \varphi$ & 0 & $\delta_{j} / \varphi$ \\
$\tilde{\omega}_{i}=w_{i}^{+}$ & $\left(\delta_{i}-\lambda_{j}\right) / \varphi$ & $\delta_{i} / \varphi$ & $\left(\delta_{i}+\delta_{j}\right) / \varphi$
\end{tabular}

Proof: Since we assume $\tilde{\Sigma}>0$, it is sufficient to show that $\tilde{\omega}$ satisfies the optimality condition (9) with $\Sigma=\tilde{\Sigma}$ and $\omega=\tilde{\omega}$. From condition (6), we have $e^{\prime} \omega=1$, so that by the transformation (12), it follows that

$\varphi \tilde{\Sigma} \tilde{\omega}=\varphi \sum \tilde{\omega}+(\delta-\lambda)+e(\delta-\lambda)^{\prime} \tilde{\omega}$.

From conditions (7) and (8), we have $\delta_{i}\left(w_{i}^{+}-\tilde{\omega}_{i}\right)=0$ and $\lambda_{i}\left(\tilde{\omega}_{i}-w_{i}^{-}\right)=0$, which implies $\delta^{\prime} \tilde{\omega}=\delta^{\prime} w^{+}$and $\lambda^{\prime} \tilde{\omega}=\lambda^{\prime} w^{-}$. Then, by (13) we have

$\varphi \tilde{\Sigma} \tilde{\omega}=\varphi \Sigma \tilde{\omega}+(\delta-\lambda)+e \delta^{\prime} w^{+}-e \lambda^{\prime} w^{-}$.

Now, from condition (5) and by (14), it follows that

$\varphi \tilde{\Sigma} \tilde{\omega}=\varphi \sigma_{M}^{2} \beta+r+\left(\tilde{\lambda}_{0}+\delta^{\prime} w^{+}-\lambda^{\prime} w^{-}\right) e$.

Finally, by (15) and condition (9), taking $\Sigma=\tilde{\Sigma}$ and $\omega=\tilde{\omega}$, we have

$$
\lambda_{0}=\tilde{\lambda}_{0}+\delta^{\prime} w^{+}-\lambda^{\prime} w^{-}
$$

showing that solution $\tilde{\omega}$ solves the unconstrained problem with $\Sigma=\tilde{\Sigma}$, completing the proof.

From Proposition 1, we have that constructing the constrained problem from $\Sigma$ is equivalent to con- structing the unconstrained problem from (12). The elements of the matrix $\tilde{\Sigma}$ can be written as $\tilde{\Sigma}_{i, j}=\Sigma_{i, j}+\Delta_{i, j}$ (as in Roncalli, 2011), where the perturbation $\Delta_{i, j}$ is specified in Table 1 , considering the conditions (7) and (8). Notice that $\Delta_{i, j}$ is negative (positive) when one optimized weight $\tilde{\omega}_{i}$ reaches its lower (upper) bound, whereas the second weight $\tilde{\omega}_{j}$ does not reach its upper (lower) bound. On the other hand, it is null when the optimized weights do not reach the lower and upper bounds. Moreover, notice that lower (upper) bounds have a negative (positive) impact on the volatility, that is $\tilde{\sigma}_{i}=\sqrt{\sigma_{i}^{2}+\Delta_{i, j}}$. Notice that imposing weights constraints is equivalent to changing the original covariance matrix $\Sigma$.

In the following, we illustrate the impact of portfolio weights constraints through a numerical example, considering an EIT portfolio composed of five assets (the parameters are presented in Table 2). The column $\omega^{*}$ shows the optimal solution for the unconstrained problem $\left(\omega \in \mathfrak{R}^{5}\right)$, calculated by (11), with $\mu_{M}=0.75 \%, \sigma_{M}^{2}=2.5 \%$ and $\varphi=1.0$. This portfolio has one short position (on the third asset) and four long positions. Now, if we impose that the weights are between $w_{j}^{-}=10 \%$ and $w_{j}^{+}=30 \%$ (e.g., $0.10 \leq \omega_{i} \leq 0.30$ ), we obtain the results presented in Table 3. The column $\tilde{\omega}$ shows the weights for the constrained EIT

Table 2. Market parameters and optimal solution for the unconstrained EIT problem $\left(\omega^{*}\right)$

\begin{tabular}{|c|c|c|c|c|c|c|c|c|}
\hline Assets & $r(\%)$ & $\beta$ & $\omega^{*}(\%)$ & \multicolumn{5}{|c|}{ Covariance matrix $\Sigma(\%)$} \\
\hline 1 & 3.29 & 1.36 & 59.93 & 4.81 & 0.58 & 0.64 & 0.31 & 0.81 \\
\hline 2 & 1.88 & 0.91 & 7.66 & 0.58 & 2.42 & 0.48 & 0.18 & 0.39 \\
\hline 3 & 1.83 & 0.88 & -3.04 & 0.64 & 0.48 & 1.35 & 0.21 & 0.43 \\
\hline 4 & 2.50 & 0.53 & 1.07 & 0.31 & 0.18 & 0.21 & 3.50 & 0.27 \\
\hline 5 & 2.10 & 1.12 & 34.39 & 0.81 & 0.39 & 0.43 & 0.27 & 2.60 \\
\hline
\end{tabular}


Table 3. Results for the constrained EIT problem, with weights constraints $0.10 \leq \omega_{i} \leq 0.30$

\begin{tabular}{|c|c|c|c|c|c|c|c|c|}
\hline Assets & $\tilde{\omega}(\%)$ & $\lambda$ & $\delta$ & \multicolumn{5}{|c|}{ Shrinkage covariance matrix $\tilde{\Sigma}(\%)$} \\
\hline 1 & 30.00 & 0.000000 & 0.013772 & 7.56 & 1.96 & 2.02 & 1.51 & 2.50 \\
\hline 2 & 14.91 & 0.000000 & 0.000000 & 1.96 & 2.42 & 0.48 & 0.00 & 0.70 \\
\hline 3 & 15.09 & 0.000000 & 0.000000 & 2.02 & 0.48 & 1.35 & 0.03 & 0.74 \\
\hline 4 & 10.00 & 0.001793 & 0.000000 & 1.51 & 0.00 & 0.03 & 3.23 & 0.40 \\
\hline 5 & 30.00 & 0.000000 & 0.003142 & 2.50 & 0.70 & 0.74 & 0.40 & 3.23 \\
\hline
\end{tabular}

problem (1)-(4) calculated using MATLAB function fmincon. It is easy to see that the solution $\tilde{\omega}$ can be obtained from (11) with $\Sigma=\tilde{\Sigma}$. Moreover, notice that the first and fifth assets reached the upper bounds, whereas the fourth asset reached its lower bound (the second and third assets did not reach the lower and upper bounds). Finally, notice that the implied volatility of the first and fifth assets increased $\left(\tilde{\sigma}_{1}>\sigma_{1}\right.$ and $\left.\tilde{\sigma}_{5}>\sigma_{5}\right)$, whereas the implied volatility of the fourth asset decreased $\left(\tilde{\sigma}_{4}<\sigma_{4}\right)$, as established in Table 1 .

\subsection{The effect of the weights constraints}

As presented in Jagannathan and Ma (2003), for the case of an unconstrained global minimum variance portfolio, an asset tends to receive low portfolio weight at the optimum if it has higher covariances with other assets. Likewise, an asset with low covariances with other assets tends to receive high weights. On the other hand, when we impose $\omega>0$, the variance of an asset $i$ and its covariances with other assets are reduced, so that the asset $i$ tends to receive high weight even if it has higher variance and higher covariances with other assets. Similarly, imposing $\omega_{i} \leq w_{i}^{+}$, the variance of the asset $i$ and its covariances with other assets are increased, so that the asset $i$ tends to receive low portfolio weight even if it has lower variance and lower covariances with other assets.

In the following, we show that the previous findings do apply to a special case of the EIT problem (1)-(4), in which $r_{i}=r_{0}$ and $\beta_{i}=\beta_{0}$ (or, equivalently, $r_{i}+\varphi \sigma_{M}^{2} \beta_{i}=c$ ). In this case, from (9), we have the following condition

$$
\varphi(\Sigma \omega)_{i}=c_{0}, i=1, \ldots, n,
$$

where $r_{0}+\varphi \sigma_{M}^{2} \beta_{0}+\lambda_{0}=c_{0}$. Let us consider that asset $i$ tends to have higher covariances with other assets, so that the $i$-th row of $\Sigma$ tends to have larger elements than other rows. Therefore, from condition (17), to achieve optimality, it is necessary to reduce asset $i$ 's portfolio weight $\left(\omega_{i}\right)$, so that the asset $i$ may even have negative weight if its covariances with other assets are sufficiently high. Thus, an asset tends to receive low weight at the optimum if it has higher covariances with other assets. Likewise, if an asset has low covariances with other assets, it tends to receive high portfolio weights. Now, let us consider that we impose the lower bonds $\left(w^{-}\right)$. From Proposition 1, we have that constructing this constrained problem from $\Sigma$ is equivalent to constructing the unconstrained problem from $\tilde{\Sigma}=\Sigma-\left(e \lambda^{\prime}+\lambda e^{\prime}\right) / \varphi$, so that, for asset $i$, its covariances with other assets are reduced by $\left(\lambda_{i}+\lambda_{j}\right) / \varphi$ and its variance by $2 \lambda_{i}$. Similarly, when upper bonds $\left(w^{+}\right)$are considered, we have from Proposition 1 that constructing the upper-bound constrained problem from $\Sigma$ is equivalent to constructing the unconstrained problem from $\tilde{\Sigma}=\Sigma+\left(e \delta^{\prime}+\delta e^{\prime}\right) / \varphi$, so that, for asset $i$, its covariances with other assets are increased by $\left(\delta_{i}+\delta_{j}\right) / \varphi$ and its variance by $2 \delta_{i}$. Thus, as we saw before, the weight of the asset $i$ tends to increase when we impose $\omega_{i} \geq w_{i}^{-}$ and tends to decrease when we impose $\omega_{i} \leq w_{i}^{+}$.

As we have shown before, if we consider $\left(r_{i}+\varphi \sigma_{M}^{2} \beta_{i}\right)=c$, then the effects of weights constraints on the EIT problem are the same as the effect on the global minimum variance portfolio problem (studied in Jagannathan \& Ma, 2003). However, it is not true in general case. In fact, how we can see from (9), we have $\varphi(\Sigma \omega)_{i}=\lambda_{0}+\left(r_{i}+\varphi \sigma_{M}^{2} \beta_{i}\right)$, so that the effects of imposing lower $\left(w_{i}^{-}\right)$and upper $\left(w_{i}^{+}\right)$bounds on the weights of the EIT portfolio depend on the 
Table 4. Solutions for the unconstrained EIT problem, $\omega^{*}$, for the constrained EIT problem with $0.1 \leq \omega_{i} \leq 0.3, \tilde{\omega}$, and for the unconstrained EIT problem with $r_{i}=0.02$ and $\beta_{i}=1.0, \bar{\omega}$

\begin{tabular}{|c|c|c|c|c|c|c|c|c|c|c|}
\hline Assets & $r(\%)$ & $\beta$ & $\omega^{*}(\%)$ & $\tilde{\omega}(\%)$ & $\bar{\omega}(\%)$ & \multicolumn{5}{|c|}{ Covariance matrix $\Sigma(\%)$} \\
\hline 1 & 3.29 & 1.36 & 59.93 & 30.00 & 4.59 & 4.81 & 0.58 & 0.64 & 0.31 & 0.81 \\
\hline 2 & 1.88 & 0.91 & 7.66 & 14.91 & 19.37 & 0.58 & 2.42 & 0.48 & 0.18 & 0.39 \\
\hline 3 & 1.83 & 0.88 & -3.04 & 15.09 & 41.49 & 0.64 & 0.48 & 1.35 & 0.21 & 0.43 \\
\hline 4 & 2.50 & 0.53 & 1.07 & 10.00 & 16.99 & 0.31 & 0.18 & 0.21 & 3.50 & 0.27 \\
\hline 5 & 2.10 & 1.12 & 34.39 & 30.00 & 17.56 & 0.81 & 0.39 & 0.43 & 0.27 & 2.60 \\
\hline
\end{tabular}

covariances $\sigma_{i, j}$, expected return $r_{i}$ and beta $\beta_{i}$. Considering the example presented in subsection 2.1, Table 4 shows the solutions for the unconstrained problem, $\omega^{*}$, for the constrained problem with $0.1 \leq \omega_{i} \leq 0.3, \tilde{\omega}$, and for the unconstrained problem with $r_{i}=0.02$ and $\beta_{i}=1.0$, $\bar{\omega}$. Notice that the weights $\bar{\omega}_{i}$ have the same behavior when compared to global minimum variance portfolio (an asset tends to receive low (high) weight at the optimum if it has higher (lower) variances with other assets), since minimizing the function (1) is equivalent to minimizing only the term $(\varphi / 2) \omega^{\prime} \Sigma \omega$. On the other hand, the unconstrained portfolio $\omega^{*}$ does not follow this behavior, since the weights will depend on the term $\left(r_{i}+\varphi \sigma_{M}^{2} \beta_{i}\right)$ and the covariances between the assets (notice now that the problem aims at minimizing the tracking error and at the same time maximizing the excess return).

\section{ASSET ALLOCATION EXAMPLE}

Following a similar analysis and procedure as in Paulo et al. (2016), in this section, we study the performance of the constrained and unconstrained EIT portfolios. We consider the S\&P 500 index as the benchmark target to be tracked using a portfolio composed of the following ten stocks $(n=10)$ : APA, CAT, APC, HPQ, ABT, CHK, ALB, AAP, KR and AJG. First, we use an in-sample analysis to investigate the performance of the models. Considering a historical series of monthly return rate in the period from July 2010 to April 2016 (a sample with size $T=69$ ), the variance of the S\&P 500 index is $\sigma_{M}^{2}=0.127 \%$ (with $\mu_{M}=0.978 \%$ ) and the parameters $\beta, r$ (in percentage points) and $\Sigma$ (in percentage points) are given by

$\begin{aligned} \beta & =\left(\begin{array}{lllllllllllll}1.578 & 1.648 & 1.728 & 1.615 & 0.900 & 1.768 & 1.885 & 0.794 & 0.717 & 1.020\end{array}\right)^{\prime}, \\ r & =\left(\begin{array}{ccccccccccc}-0.353 & 0.480 & 0.540 & -0.280 & 0.860 & -0.428 & 1.053 & 1.803 & 1.939 & 0.977\end{array}\right)^{\prime}, \\ \Sigma & =\left(\begin{array}{ccccccccccc}0.917 & 0.527 & 0.701 & 0.374 & 0.098 & 0.907 & 0.540 & 0.054 & -0.039 & 0.196 \\ 0.527 & 0.663 & 0.518 & 0.402 & 0.121 & 0.504 & 0.583 & 0.188 & 0.032 & 0.218 \\ 0.701 & 0.518 & 0.886 & 0.401 & 0.142 & 0.979 & 0.507 & 0.159 & 0.072 & 0.252 \\ 0.374 & 0.402 & 0.401 & 0.967 & 0.168 & 0.394 & 0.495 & 0.140 & 0.122 & 0.245 \\ 0.098 & 0.121 & 0.142 & 0.168 & 0.254 & 0.102 & 0.130 & 0.082 & 0.123 & 0.133 \\ 0.907 & 0.504 & 0.979 & 0.394 & 0.102 & 2.473 & 0.400 & 0.138 & -0.092 & 0.271 \\ 0.540 & 0.583 & 0.507 & 0.495 & 0.130 & 0.400 & 0.909 & 0.161 & 0.107 & 0.241 \\ 0.054 & 0.188 & 0.159 & 0.140 & 0.082 & 0.138 & 0.161 & 0.474 & 0.101 & 0.150 \\ -0.039 & 0.032 & 0.072 & 0.122 & 0.123 & -0.092 & 0.107 & 0.101 & 0.353 & 0.079 \\ 0.196 & 0.218 & 0.252 & 0.245 & 0.133 & 0.271 & 0.241 & 0.150 & 0.079 & 0.229\end{array}\right) .\end{aligned}$


Let $\tilde{\omega}$ be the solution of the constrained problem (1)-(4), obtained by MATLAB function fmincon, and $\omega^{*}$ be the solution of the unconstrained problem $\left(\omega \in \mathfrak{R}^{10}\right)$, obtained from (11). Considering an EIT strategy with $\varphi=15$, and taking $w_{i}^{-}=0.03$ and $w_{i}^{+}=0.15$ (e.g., the short positions are not allowed), it follows that (in percentage points)

$$
\begin{aligned}
\tilde{\omega} & =\left(\begin{array}{lllllllllll}
3.01 & 12.17 & 3.84 & 3.01 & 14.99 & 3.00 & 14.98 & 15.00 & 15.00 & 15.00
\end{array}\right)^{\prime}, \\
\omega^{*} & =\left(\begin{array}{llllllllll}
-3.07 & 4.45 & 2.95 & -11.42 & 12.22 & 1.47 & 17.09 & 15.22 & 34.56 & 26.53
\end{array}\right)^{\prime} .
\end{aligned}
$$

Now we compare the performance of the constrained and unconstrained EIT portfolios over the time period [0;69]. Let $\tilde{V}(t)$ and $V^{*}(t)$ be the values of the EIT portfolios related to the solutions $\tilde{\omega}$ and $\omega^{*}$, respectively, written as

$\tilde{V}(t+1)=\left(1+R(t)^{\prime} \tilde{\omega}\right) \tilde{V}(t)$,

$V^{*}(t+1)=\left(1+R(t)^{\prime} \omega^{*}\right) V^{*}(t)$,

where $\quad R(t)=\left(R_{1}(t) \ldots R_{n}(t)\right)^{\prime}, \quad$ with
$t=0, \ldots, T-1 \quad$ and $\tilde{V}(0)=V^{*}(0)=V_{0}$. Similarly, let $V^{b}(t)$ be the position value of the position associated with the S\&P 500 index, given by we consider an in-sample analysis.

$$
V^{b}(t+1)=\left(1+R_{M}(t)\right) V^{b}(t),
$$

where $R_{M}(t)$ is the return of the S\&P 500 index with $V^{b}(0)=V_{0}^{b}$. Taking $V_{0}=V_{0}^{b}=100$, we present in Figure 1 the cumulative monthly portfolio values over the time period $[0 ; 69]$.

Since we want to track the S\&P 500 index with positive mean excess return (e.g., EIT strategy), we can observe from Figure 1 that (on average) the constrained EIT portfolio $(\tilde{\omega})$ performs better than the unconstrained EIT portfolio $\left(\omega^{*}\right)$. Specifically, the portfolio $\tilde{\omega}$ has a positive excess return rate $(P E R)$ of $P E R_{\tilde{\omega}}=85.51 \%$ and the portfolio $\omega^{*}$ has a positive excess return rate of $P E R_{\omega^{*}}=97.10 \%$, over the period $[0 ; 69]$, while their root mean square is given by $R M S_{\tilde{\omega}}=7.00$ and $R M S_{*}=49.25$, respectively. Using the ratio $I R=\stackrel{\oplus}{P} E R / R M S$ as a performance measure, we can notice that $I R_{\tilde{\omega}}=12.21>I R_{\omega^{*}}=1.97$, showing that the portfolio $\tilde{\omega}$ performs better than the portfolio $\omega^{*}$ when

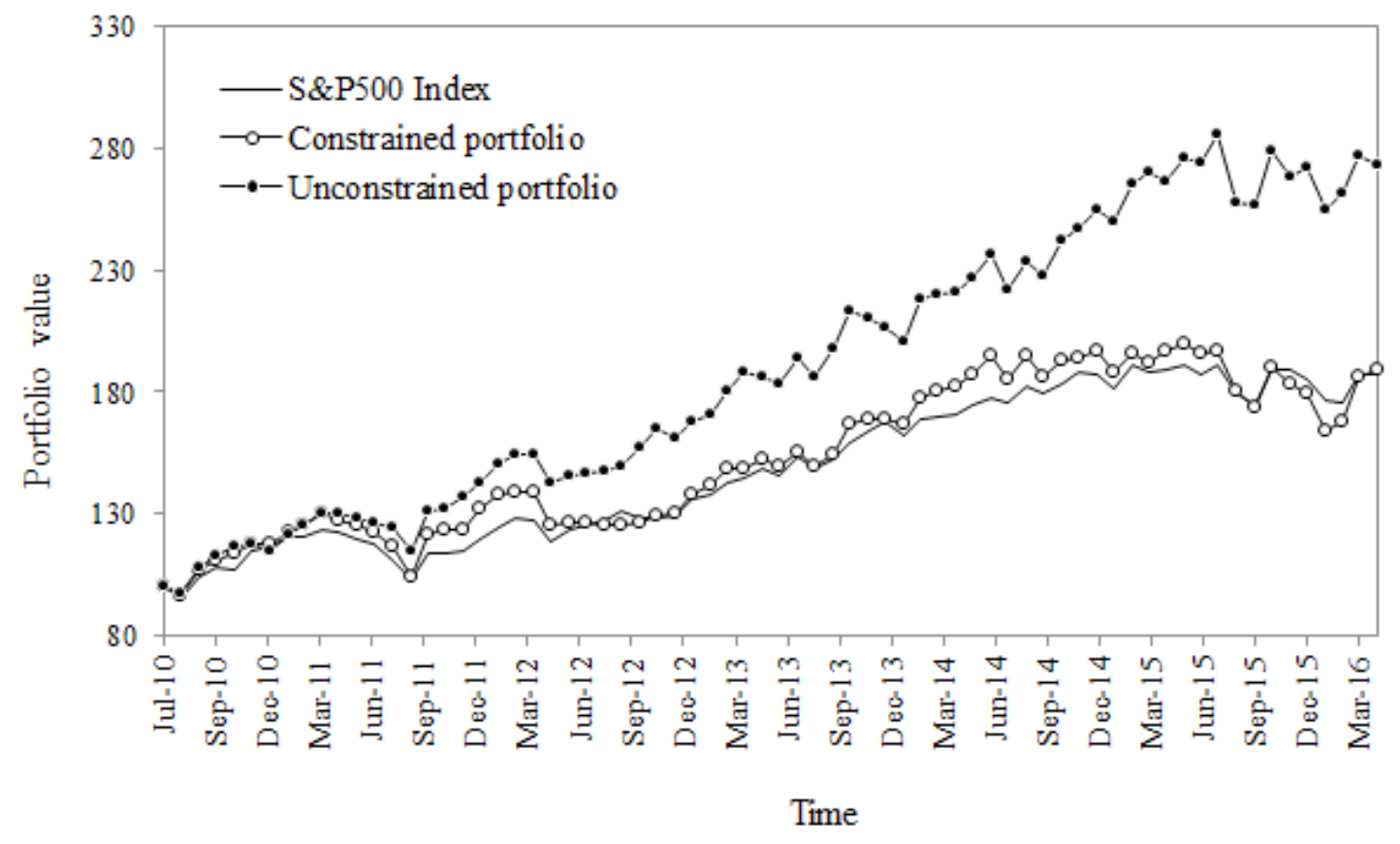

Figure 1. Cumulative monthly portfolio values, obtained by the constrained EIT portfolio $\tilde{\omega}$ and by the unconstrained EIT portfolio $\omega^{*}$, considering an in-sample analysis 


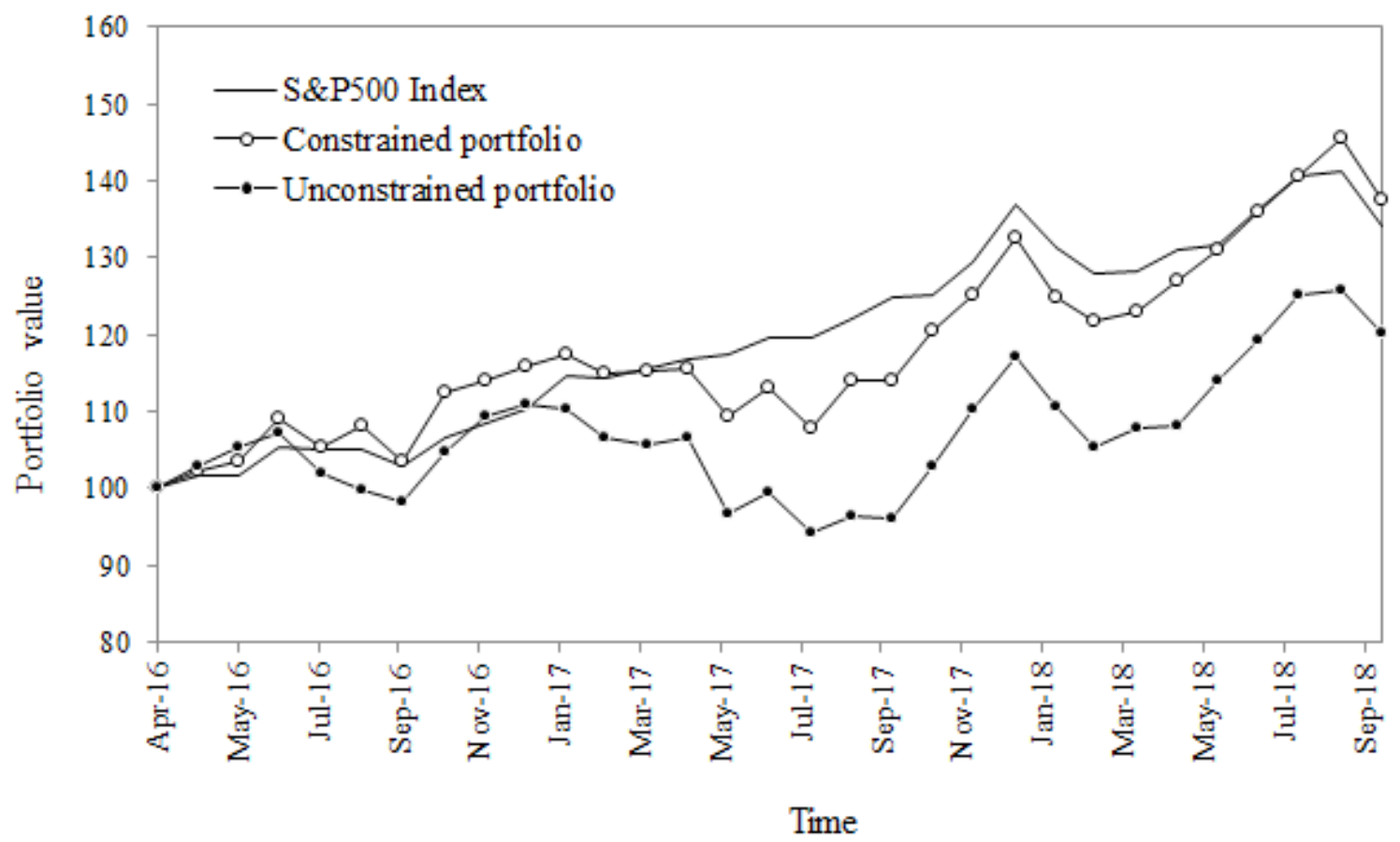

Figure 2. Cumulative monthly portfolio values, obtained by the constrained EIT portfolio $\tilde{\omega}$ and by the unconstrained EIT portfolio $\omega^{*}$, considering an out-of-sample analysis

In the analysis presented above, we used the data from July 2010 to April 2016 to estimate the parameters of the model and to measure its performance based on an in-sample criterion (using the indicator $I R$ ). In the following, we present a similar analysis considering an out-of-sample performance, where the period from April 2016 to October 2018 was considered (a sample with size $T=30$ ). Figure 2 shows the cumulative monthly portfolio values over the time period $[0 ; 30]$, in which we can see that the constrained and unconstrained portfolios perform poorly in general (with $P E R_{\tilde{\omega}}=33 \%$ and $P E R_{\sigma^{*}}=17 \%$ ). This result occurs due to the significant changes in market parameter values during out-of-sample period (compared to the insample period). In this situation, the performance of the portfolios can be improved if we rebalance them periodically. Figure 3 shows the cumulative monthly portfolio values in out-of-sample period, in which we rebalance the weights $\tilde{\omega}$ and $\omega^{*}$ monthly. For this propose, we reestimate the parameters $r$ and $\mu_{M}$ using a moving average criteria (with sample size $T=30$ ) and the parameters $\Sigma$ and $\sigma_{M}^{2}$ using the EWMA method with decay factor $\lambda=0.75$ (for more details about these methods, see Alexander, 2008, Volume 2, Chapter 3 ). As we can see from Figure 3, in general, the rebalanced portfolios perform better than the origi- nal portfolios (without rebalancing). Specifically, we have $P E R_{\tilde{\omega}}=73.33 \%, P E R_{\omega^{*}}=63.33 \%$, $R M S_{\tilde{\omega}}=2.89$ and $R M S_{\omega^{*}}=4.46$, such that $I R_{\tilde{\omega}}=25.37>I R_{\omega^{*}}=14.18$. Then, as in-sample analysis, the constrained EIT portfolio $(\tilde{\omega})$ also performs better than the unconstrained EIT portfolio $\left(\omega^{*}\right)$ in out-of-sample period when we consider a rebalancing investment strategy.

Finally, we remark that the performance of the unconstrained EIT portfolio $\omega^{*}$ can be improved by choosing an appropriate trade-off parameter $\varphi$, such that the previous results cannot be generalized (for both in-sample and out-of-sample cases). For example, considering the in-sample period, if we set $\varphi=93.25$ only for the unconstrained EIT portfolio, we have (in percentage points) $\omega^{*}=\left(\begin{array}{lllll}8.74 & 9.54 & -4.24 & -0.79 & 22.10\end{array}\right.$

\section{$\left.\begin{array}{lllll}2.16 & 6.92 & 7.76 & 22.73 & 25.08\end{array}\right)^{\prime}$,}

such that $P E R_{\omega^{*}}=84.06 \%$ and $R M S_{\omega^{*}}=7.00$ (equal to the $\stackrel{\omega}{R} M S_{\tilde{\omega}}$ ). Comparing to the constrained EIT portfolio $\tilde{\omega}$, we have that $I R_{\omega^{*}}=12.01 \approx I R_{\tilde{\omega}}=12.21$. Then, in this particular case, it was possible to reach a similar performance level for both portfolios by choosing an appropriate parameter $\varphi$. Although we had achieved 


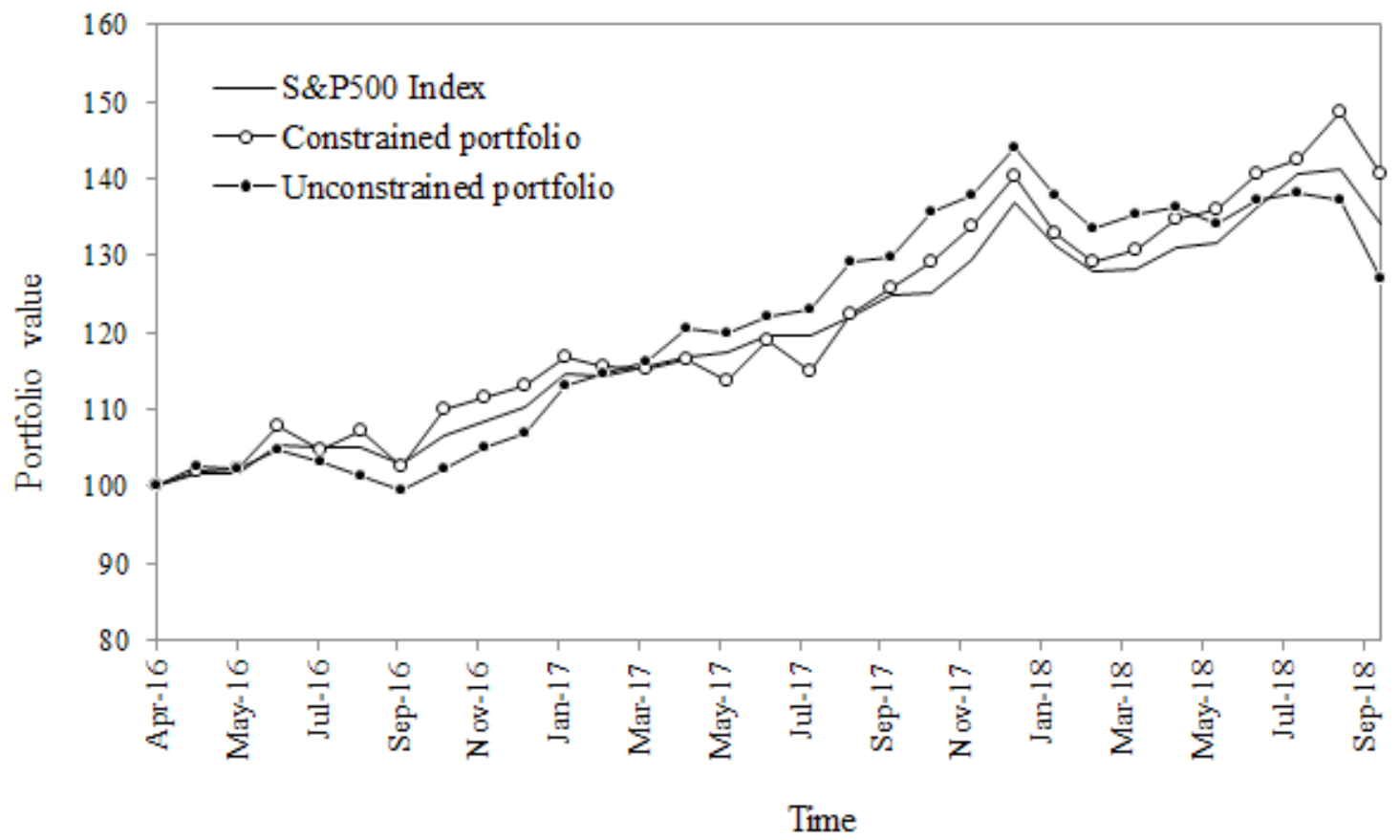

Figure 3. Cumulative monthly portfolio values, obtained by rebalancing the weights $\tilde{\omega}$ and $\omega^{*}$ during the out-of-sample period

a good performance level by setting the parameter $\varphi$, from the practical point of view, the constrained EIT problem is more appropriate to construct a EIT portfolio than the unconstrained EIT problem, since the first one allows to control the level of short and long positions, as well as to avoid the concentration risk (when a portfolio has a large exposure to an asset or few assets).

\section{FINAL REMARKS}

In this paper, we consider an uni-period mean-variance enhanced index tracking (EIT) problem with weights constraints. As theoretical result, we show that constructing the constrained EIT portfolio is equivalent to constructing the unconstrained EIT portfolio (as studied in Paulo et al., 2016), when we modify the covariance matrix in a particular way (shrinkage covariance matrix). From this equivalence, we analyze the impact of weights constraints on the covariance matrix and on the EIT portfolio. In general, the effects of weights constraints on the EIT portfolio are different from those observed in the case of global minimum variance portfolio (as studied in Jagannathan \& Ma, 2003). We present a numerical example using the S\&P 500 index as the market index to be tracked using a portfolio composed of ten stocks. We observe that the constrained EIT portfolio performs better than the unconstrained EIT portfolio. This particular result cannot be generalized, since the performance of the unconstrained EIT portfolio can be improved by choosing an appropriate parameter $\varphi$. However, we highlight that a constrained problem is more appropriate to construct a portfolio than an unconstrained problem, since the first one allows to control the level of short and long positions and avoids the concentration risk.

\section{REFERENCES}

1. Alexander, C. (2008). Market Risk Analysis: Quantitative Methods in Finance. New Jersey: Wiley.

2. Alexander, G. J., \& Baptista, A. M. (2010). Active portfolio manage- ment with benchmarking: A

frontier based on alpha. Journal of Banking and Finance, 34, 21852197, https://doi.org/10.1016/j. jbankfin.2010.02.005
3. Benidis, K., Feng, Y., \& Palomar, D. P. (2018). Sparse portfolios for high-dimensional financial index tracking. IEEE Transactions on signal processing, 66, 
155-170. https://doi.org/10.1109/ TSP.2017.2762286

4. Canakgoz, N. A., \& Beasley, J. E. (2008). Mixed-integer programming approaches for index tracking and enhanced indexation. European Journal of Operational Research, 196, 384-399. https://doi. org/10.1016/j.ejor.2008.03.015

5. DeMiguel, V., Garlappi, L., Nogales, F. J., \& Uppal, R. (2009). A generalized approach to portfolio optimization: Improving performance by constraining portfolio norms. Management Science, 55, 798-812. http://dx.doi. org $/ 10.1287 / \mathrm{mnsc} .1080 .0986$

6. Fan, J., Zhang, J., \& Yu, K. (2012). Vast portfolio selection with grossexposure constraints. Journal of the American Statistical Association, 107, 592-606. https://doi.org/ 10.1080/01621459.2012.682825

7. Filippi, C., Guastaroba, G., \& Speranza, M. G. (2016). A heuristic framework for the bi-objective enhanced index tracking problem. Omega, 65, 122-137. https://doi. org/10.1016/j.omega.2016.01.004
8. Goel, A., Sharma, A., \& Mehra, A. (2018). Index tracking and enhanced indexing using mixed conditional value-at-risk. Journal of Computational and Applied Mathematics, 335, 361-380. https://doi. org/10.1016/j.cam.2017.12.015

9. Jagannathan, R., \& Ma, T. (2003). Risk reduction in large portfolios: Why imposing the wrong constraints helps. The Journal of Finance, 58, 1651-1683. https:// doi.org/10.1111/1540-6261.00580

10. Kim, J. H., Kim, W. C., \& Fabozzi, F. J. (2016). Portfolio selection with conservative short-selling. Finance Research Letters, 18, 363-369. https://doi.org/10.1016/j. frl.2016.05.015

11. Ledoit, O., \& Wolf, M. (2004). A well-conditioned estimator for large-dimensional covariance matrices. Journal of Multivariate Analysis, 88, 365-411. https://doi.org/10.1016/S0047259X(03)00096-4

12. Pantaleo, E., Tumminello, M., Lillo, F., \& Mantegna, R. N. (2011). When do improved covariance matrix estimators enhance portfolio optimization? An empirical comparative study of nine estimators. Quantitative Finance, 11, 1067-1080. https://doi.org/10.1080 /14697688.2010.534813

13. Paulo, W. L., Oliveira, E. M., \& Costa, O. L. V. (2016). Enhanced index tracking optimal portfolio selection. Finance Research Letters, 16, 93-102. https://doi. org/10.1016/j.frl.2015.10.005

14. Roll, R. (1992). A mean/variance analysis of tracking error. The Journal of Portfolio Management, 18, 13-22. https://doi.org/10.3905/ jpm.1992.701922

15. Roncalli, T. (2011). Understanding the Impact of Weights Constraints in Portfolio Theory. Retrieved from https://ssrn.com/abstract=1761625

16. Sant'Anna, L. R., Filomena, T. P., \& Caldeira, J. F. (2017). Index tracking and enhanced indexing using cointegration and correlation with endogenous portfolio selection. The Quarterly Review of Economics and Finance, 65, 146-157. https:// doi.org/10.1016/j.qref.2016.08.008 